\title{
POSITION DEPENDENT HEAVY ATOM EFFECT IN PHYSICAL TRIPLET QUENCHING BY ELECTRON DONORS
}

\author{
UIrich STEINER and Gerhard WINTER \\ Institut für Physikalische Chemie der Universität Stuttgart, \\ D-7000 Stuttgart 80, Germany
}

\begin{abstract}
The radical yields and rate constants in the quenching reaction of thionine triplet with the complete series of monohalogen substituted anilines as electron donors were determined by flash spectroscopy. Whereas the quenching rate constants show little and unsystematic variation, the radical yields decrease with increasing spin-orbit coupling constant of the halogen substituent. This effect is very sensitive to the position of the halogen in the donor. The results are explained in terms of a heavy atom effect on the intersystem crossing rate constant in a triplet exciplex.
\end{abstract}

\section{Introduction}

In electron transfer (ET) reactions excited triplet states are often observed to be physically quenched to some extent. As we have found [1], with heavy atoms in the electron donor the physical quenching process may become dominant in accord with the general observation that intersystem crossing (ISC) processes are enhanced by heavy atoms. Two mechanisms are conceivable for this effect:

(a) the heavy atom quenching may be independent of the ET reaction (external heavy atom effect) or (b) the heavy atom may enhance an ISC route already inherent in the ET reaction.

For ET reactions with the excited pyrene singlet Weller and co-workers [2] have found that two ISC mechanisms lead to fast triplet formation: spin-orbit coupling in a primarily formed exciplex and hyperfine coupling in the geminate radical pair which is formed by dissociation of the exciplex. The hyperfine mechanism of triplet formation has also been investigated by Michel-Beyerle et al. [3] . Of these two mechanisms only the exciplex ISC would be expected to be enhanced by heavy atoms. Indeed, for a fluorescing exciplex [4] and several phosphorescing charge transfer complexes [5], heavy atom effects on ISC rate constants have been reported.
To study the mechanism underlying the triplet to ground state deactivation by electron donors in greater detail, we investigated the quenching reaction of the thionine triplet ${ }^{3} \mathrm{TH}^{+}$with the complete series of monohalogen substituted anilines as donors. As these molecules have only slightly different oxidation potentials we expected conclusive information as to the nature of the reactions, contributing to the triplet quenching constant: if a reaction involving ISC contributes to the first step of the quenching, the quenching constant should be significantly enhanced with increasing nuclear charge of the halogen substituent (external heavy atom effect), whereas if the quenching consists entirely of a spin conserving ET step, no significant variation of the quenching constant would be expected with this series of donors.

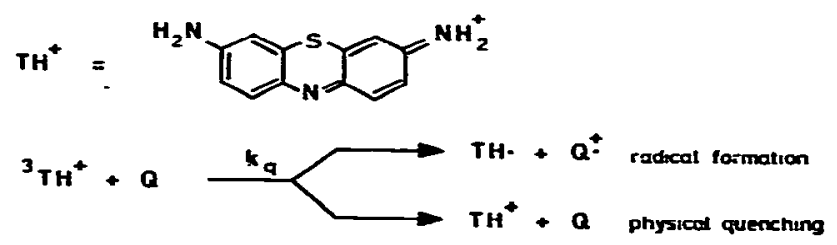

\section{Experimental}

The apparatus and techniques have been described 
previously [1] and hence only a brief summary will be given here. The thionine triplet ${ }^{3} \mathrm{TH}^{+}$in methanol as solvent was produced by conventional flash excitation and the triplet decay as well as the formation of semithionine radicals TH', produced in the quenching reaction, was followed by kinetic absorption spectroscopy. The solutions were buffered at a pH of $8.6(0.015 \mathrm{M}$ phenylacetic acid, $0.005 \mathrm{M}$ sodium methylate [6]), so that the semithionine radicals were rapidly protonated. This procedure was used since the protonated semithionine has an intense absorption peak in the long wavelength region $\left(\lambda_{\max }=780 \mathrm{~nm}\right)$ which is not overlapped by the absorption of the oxidized quencher radicals. Furthermore the protonated semithionine is rather long-lived (milliseconds), so the radical yield measured about $25 \mu$ s after flash triggering is not affected by second order recombination reactions. For each donor we determined the triplet quenching constant $k_{\mathrm{q}}$ and the radical yield $\eta_{\mathrm{r}}$, defined as the fraction of radicals formed per excited triplet molecule under conditions of complete quenching. By observation of the thionine ground state repopulation during the quenching reaction it was confirmed that physical quenching was the only important quenching channei besides radical formation.

\section{Results anci discussion}

The quenching constants $k_{\mathrm{q}}$ and radical yields $\eta_{\mathrm{r}}$ obtained for the reaction of ${ }^{3} \mathrm{TH}^{+}$with aniline and its monohalogen substituted derivatives are given in table 1. Whereas the quenching rate constants show little and unsystematic variation, the radical yields decrease monotonically as the nuclear charge of the halogen increases. A marked positional dependence of this heavy atom effect is to be observed. We analyse the data in terms of two cifferent kinetic schemes:

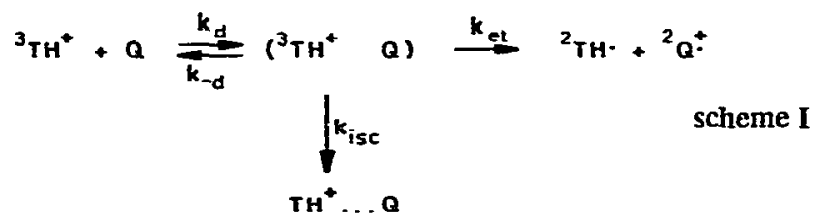

In scheme $I$ it is assumed that an encounter complex of triplet and quencher $\left({ }^{3} \mathrm{TH}^{+} \ldots \mathrm{Q}\right)$, which forms and dissociates in diffusion controlled reactions (rate constants $k_{\mathrm{d}}$ and $\left.k_{-\mathrm{d}}\right)$, has two independent reaction channels:

(a) electron transfer from $Q$ to ${ }^{3} \mathrm{TH}^{+}$(rate constant $k_{\text {et }}$ ) whereby the radicals $T H \cdot$ and $Q^{\ddagger}$ are formed,

(b) ISC of ${ }^{3} \mathrm{TH}^{+}$to the ground state (rate constant

$k_{\text {isc }}$ ), induced by the heavy atom in the quencher mole-

Table 1

Observed quenching constants $k_{\mathrm{q}}$ and radical yields $\eta_{\mathrm{r}}$, standard oxidation potentials $E_{\mathrm{O}}$ according to ref. [9], relative rate constants of electron transfer $\boldsymbol{n}_{\text {et }}$ and intersystem crossing $\boldsymbol{n}_{\text {isc }}$ from kinetic anaiysis according to scheme $I$ and scheme $I I$

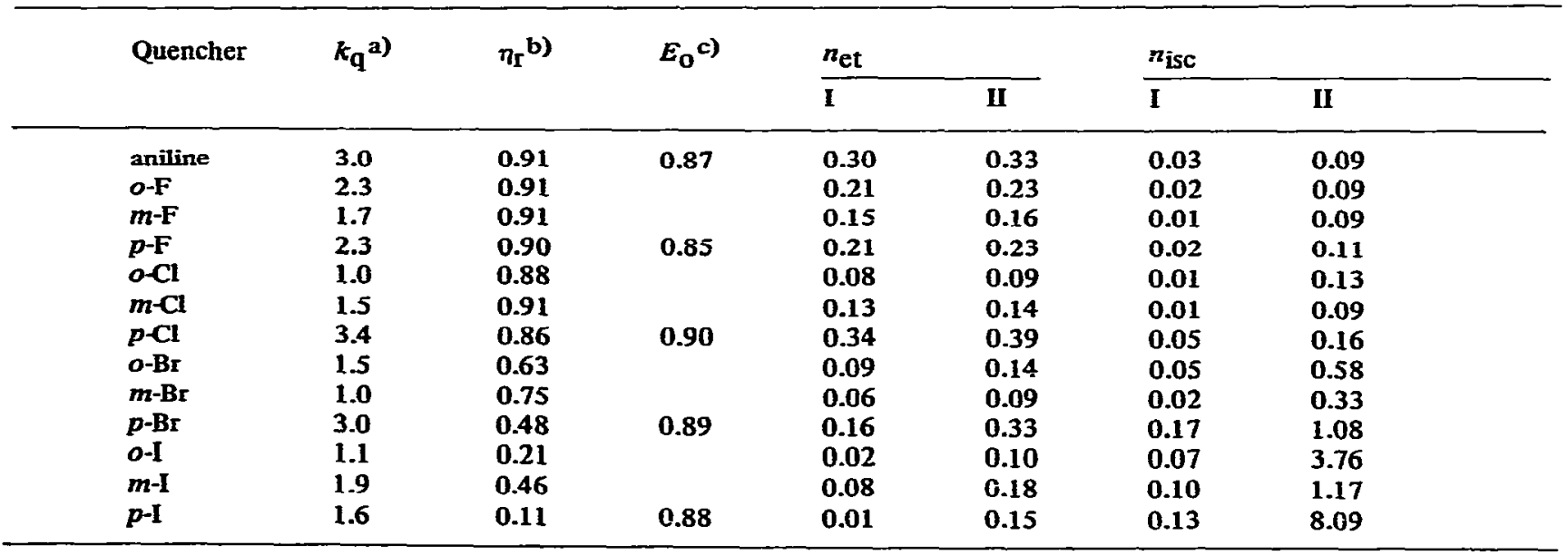

a) $10^{9} \mathrm{~s}^{-1} \mathrm{M}^{-1}$ \&. b) Relative accuracy $\pm 3 \%$. c) Volt versus SCE. 
cule (external heavy atom effect).

Within scheme I the observed quenching constant $k_{\mathbf{q}}$ and the radical yield $\eta_{\mathrm{r}}$ are given by

$k_{\mathrm{q}}=k_{\mathrm{d}}\left(k_{\mathrm{et}}+k_{\text {isc }}\right) /\left(k_{-\mathrm{d}}+k_{\mathrm{et}}+k_{\mathrm{isc}}\right)$,

$\eta_{\mathrm{r}}=k_{\mathrm{et}} /\left(k_{\mathrm{et}}+k_{\mathrm{isc}}\right)$.

From these relations we obtain relative rate constants of ET $\left(n_{\text {et }}(\mathrm{I})\right)$ and ISC $\left(n_{\text {isc }}(\mathrm{I})\right)$ :

$n_{\mathrm{et}}(\mathrm{I})=k_{\mathrm{et}} / k_{-\mathrm{d}}=\left[k_{\mathrm{q}} /\left(k_{\mathrm{d}}-k_{\mathrm{q}}\right)\right] \eta_{\mathrm{I}}$,

$n_{\text {isc }}(\mathrm{I})=k_{\text {isc }} / k_{-\mathrm{d}}=\left[k_{\mathrm{q}} /\left(k_{\mathrm{d}}-k_{\mathrm{q}}\right)\right]\left(1-n_{\mathrm{r}}\right)$.

In these expressions $k_{\mathrm{d}}$ is the rate constant of the diffusion controlled encounter reaction, calculated by $k_{\mathrm{d}}=$ $8 R T / 3000 \eta$ as $1.2 \times 10^{10} \mathrm{~s}^{-1} \mathrm{M}^{-1} \ell$.

$$
\begin{aligned}
& { }^{3} \mathrm{TH}^{+}+Q \stackrel{\mathrm{k}_{-d}}{\underset{k_{-d}}{\rightleftarrows}}\left({ }^{3} \mathrm{TH}^{+}, Q\right) \\
& \stackrel{k_{\text {ef }}}{\longrightarrow}{ }^{3}\left(\mathrm{TH} . \mathrm{Q}^{+}\right) \stackrel{k_{\mathrm{fr}}}{\longrightarrow}{ }^{2} \mathrm{TH} .{ }^{2} \mathrm{Q}^{*} \\
& k_{\text {isc }} \\
& \mathrm{TH}^{*} \ldots \mathrm{O}
\end{aligned}
$$

scheme II

According to scheme II the rate constant of triplet quenching is determined by the ET step (rate constant $k_{\mathrm{e}}$ ) by which a primary ET product denoted as 3 (TH... $Q^{+}$) is formed from the encounter complex. The species ${ }^{3}\left(\mathrm{TH}\right.$... $\left.\mathrm{Q}^{+}\right)$will be called a triplet exciplex even though the electron transfer may be nearly complete. We use the term exciplex to emphasize the fact that there is some electronic interaction betwean the radical species $\mathrm{TH}^{-}$and $\mathrm{Q}^{\ddagger}$ due to their close proximity immediately after their formation, rather than to describe a fixed conformation. The triplet exciplex can (a) dissociate to form the free radicals (rate constant $\left.k_{\mathrm{fr}}\right)$,

(b) undergo ISC to the ground state (rate constant $k_{\text {isc }}$ ). Within scheme II the observed quenching constant $\boldsymbol{k}_{\mathrm{q}}$ and the radical yield $\boldsymbol{\eta}_{\mathrm{r}}$ are given by:

$k_{\mathrm{q}}=\left[k_{\mathrm{et}}\left[k_{\mathrm{et}} /\left(k_{\mathrm{et}}+k_{-\mathrm{d}}\right)\right] k_{\mathrm{d}}\right.$,

$\eta_{\mathrm{r}}=k_{\mathrm{fr}} /\left(k_{\mathrm{isc}}+k_{\mathrm{fr}}\right)$.

From these we obtain the relative rate constants of ET $\left(n_{\mathrm{et}}(\mathrm{II})\right)$ and ISC $\left(n_{\text {isc }}\right.$ (II)):

$$
\begin{aligned}
& n_{\mathrm{et}}(\mathrm{II})=k_{\mathrm{et}} / k_{-\mathrm{d}}=k_{\mathrm{q}} /\left(k_{\mathrm{d}}-k_{\mathrm{q}}\right), \\
& n_{\mathrm{icc}}(\mathrm{I})=k_{\mathrm{isc}} / k_{\mathrm{fr}}=\left(1-\eta_{\mathrm{r}}\right) / \eta_{\mathrm{r}} .
\end{aligned}
$$

\subsection{Relative rate constants of electron transfer}

As was shown by Rehm and Weller [7] for ET reactions with excited singlet states and by Vogelmann et al. [8] for ET reactions with dye triplets, the rate constants are largely determined by the free enthalpy $(\Delta G)$ of the ET reaction. With equal $\Delta G$ the rate constants usually do not differ more than by a factor of 3-4. As in the donor-acceptor pairs applied in this work the acceptor is always the same, $\Delta G$ of the ET reaction should vary with the standard oxidation potential of the donor. In table 1 are given the corresponding values for aniline and its $p$-substituted halogen derivatives, according to the most recent literature available [9] . The difference between any two of them does not exceed $0.05 \mathrm{~V}$, a range which also applies to the results of earlier work [10], where the ortho and para derivatives were considered as well. Due to this small variation of the oxidation potentials one should expect no great differences in the ET rate constants. As table 1 shows:

$$
0.01 \leqslant n_{\mathrm{et}}(\mathrm{I}) \leqslant 0.34, \quad 0.09 \leqslant n_{\mathrm{et}}(\mathrm{II}) \leqslant 0.39 \text {. }
$$

Whereas scheme I leads to an unreasonably large scatter of the ET rate constants ( $\max . / \mathrm{min} .=34$ ), scheme II yields values in accordance with the general observations $(\max . / \min .=4.3)$.

\subsection{Relative rate constants of intersystem crossing}

The ISC constants $n_{\text {isc }}$ are expected to display the heavy atom effect clearly. The $\boldsymbol{n}_{\text {isc }}$ constants evaluated according to scheme $I$ are enhanced significantly only in $\boldsymbol{p}$-bromoaniline and the iodoanilines. However, the heaviest atom is not the most efficient! In the $n_{\text {isc }}$ constants evaluated according to scheme II the heavy atom effect increases monotonically with the spin-orbit coupling constant of the halogen substituent. Thus, again, only the constants calculated according to scheme II are physically reasonable.

To discuss the heavy atom effect on the ISC constant of the triplet exciplex ${ }^{3}$ (TH... $\left.Q^{+}\right)$in a more quantitative way we define the heavy atom effect as 
Table 2

Comparison of various heavy atom effects (relative values, $(\mathrm{Br})=100$ )

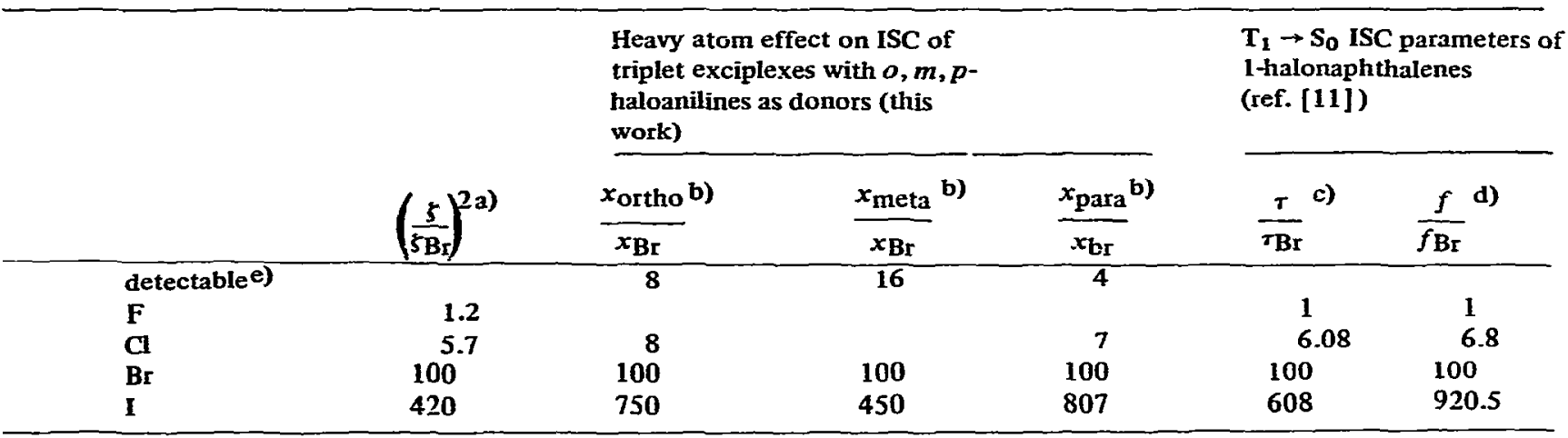

a) 5 atomic spin-orbit coupling constant, ref. [11].

b) $\boldsymbol{x}=\boldsymbol{n}_{\text {isc }}$ (haloaniline) $-\boldsymbol{n}_{\text {isc }}$ (aniline), $n_{\text {isc }}$ values calculated according to scheme II.

c) $\tau$ phosphorescence lifetime. d) $f$ integrated $S_{0} \rightarrow T_{1}$ absorption intensity.

e) Limited by relative accuracy of radical yield measurement of $\pm 3 \%$.

$\boldsymbol{x}=\boldsymbol{n}_{\text {isc }}($ haloaniline $)-\boldsymbol{n}_{\text {isc }}$ (aniline)

where we use the $\boldsymbol{n}_{\text {isc }}$ values calculated according to scheme II. A comparison of $\boldsymbol{x}$ for different halogen atoms in the same position is given in table 2 together with the squares of the halogen atom spin-orbit coupling constants $\zeta$. The correlation of $x$ with $\zeta^{2}$ is obvious. For comparison data of the intramolecular heavy atom effect on the $T_{1}-S_{0}$ transition probability in 1-halonaphthalenes [11] are also given in table

2. As can be seen the intramolecular heavy atom effect is quite analogous to what we calculated for the hypothetical species ${ }^{3}\left(\mathrm{TH} . . . \mathrm{Q}^{+}\right)$.

The dependence of $x$ on the position of the same halogen atom in the donor is shown in table 3. For reasons of accuracy only the bromo- and iodo-deriva-

Table 3

Dependence of heavy atom effect on heavy atom position (relative values, $p$-derivative of same heavy atom $=100$ )

\begin{tabular}{lccc}
\hline & ${\frac{x_{\mathrm{Br}}}{x_{\mathrm{p}}}}^{\text {a) }}$ & $\frac{x_{1}{ }^{\text {a) }}}{x_{\mathrm{p}}}$ & $\frac{c_{2}^{2} \text { b) }}{c_{\mathrm{p}}}$ \\
\hline ortho & 49 & 45 & 59 \\
meta & 24 & 13 & 10.5 \\
para & 100 & 100 & 100 \\
\hline
\end{tabular}

a) $x=n_{\text {isc }}$ (haloaniline) $-n_{\text {isc }}$ (aniline), $n_{\text {isc }}$ values calculated according to scheme II.

b) $C$ HMO coefficient of halogen adjacent $C$ atom in the highest filled aniline orbital. tives are considered. The position dependence appears to be roughly the same for different heavy atoms and correlates with the $\pi$-electron spin density (HMO calculation) at the halogen adjacent carbon in the aniline monopositive ion.

From tables 2 and 3 it may be inferred that, to a first approximation, the heavy atom effect can be factorized in a factor proportional to $\xi^{2}$ and a factor $f_{\text {pos }}$ depending only on the position and not on $\xi$, viz.

$x \propto \xi^{2} f_{\text {pos }}$ -

\subsection{Theoretical treatment of the heavy atom effect}

The empirical relation (10) can be rationalized if one regards the process ${ }^{3}\left(\mathrm{TH} . . \mathrm{Q}^{+}\right) \rightarrow{ }^{1}\left(\mathrm{TH}^{+} \ldots \mathrm{Q}\right)_{0}$ as a radiationless transition, the rate of which is proportional to the square of the matrix element of the spinorbit coupling hamiltonian between initial and vibrationally excited final state of equal energy:

$$
\left.\left.k_{\text {isc }} \propto\left|<^{3}\left(\mathrm{TH} \ldots \mathrm{Q}^{+}\right)\right| H_{\text {so }}\right|^{1}\left(\mathrm{TH}^{+} \ldots \mathrm{Q}\right)_{0, \mathrm{vib}}\right\rangle\left.\right|^{2} \text {. }
$$

In the following we shall consider only the electronic part of this matrix element for a particular triplet substate, e.g. the one with spin function $\left|\alpha_{1} \alpha_{2}\right\rangle$. The $z$ direction will be defined orthogonal to the molecular plane of the aniline part of the complex. Denoting the singly occupied $\pi$-orbitals of acceptor and donor as $a$ and $d$, respectively, the contribution of the heavy atom is given by the integral over molecular orbitals [12] : 


$$
k_{\text {isc }} \propto\left|\left\langle a\left|H_{x, \text { ha }}^{\prime}+\mathrm{i} H_{y, \text { ha }}^{\prime}\right| d\right\rangle\right|^{2},
$$

where $H_{x, \text { ha }}^{\prime}$ and $H_{y, \text { ha }}^{\prime}$ are the $x$ and $y$ components of the space part of the spin-orbit hamiltonian due to the heavy atom centre. As was shown by Friedrich et al. [13] only the one centre integrals are important in calculating the heavy atom effect. Therefore the molecular orbitals $a$ and $d$ can be projected onto the heavy atom $p$ orbitals [14], which yields approximately:

$k_{\mathrm{isc}} \propto\left(S_{a, \mathrm{p} x, \mathrm{ha}}^{2}+S_{a, \mathrm{p} y, \mathrm{ha}}^{2}\right) c_{d, \mathrm{ha}}^{2} \zeta_{\mathrm{ha}}^{2}$.

$S_{a, \mathrm{p}_{x} \text {, ha }}$ and $S_{a, \mathrm{p}_{y, \text { ha }}}$ are the overlap integrals of the acceptor MO $a$ with the AO's $p_{x}$ and $p_{y}$, respectively, of the heavy atom; $c_{d, \text { ha }}$ is the MO coefficient of $\mathrm{p}_{z, h a}$ in the donor MOd.

As a simple HMO treatment of the fluoro-, chloroand bromo-aniline shows, $c_{d, \text { ha }}$ is essentially the same for all halogens and depends only on the position, in the same way as the coefficient of the halogen adjacent carbon in the highest filled orbital of aniline. If it is assumed that the sum of squares of the overlap integrals is not very different for $\mathrm{Br}$ and $\mathrm{I}$, and that it does not depend significantly on the halogen position in the donor, the theoretical expression (13) has just the form of the empirical relation (10).

\section{Conclusions}

This paper reports the observation of a position dependent heavy atom effect on the intersystem crossing process, following the electron transfer from halogenanilines tc thionine triplet. The effect obeys a simple rule and provides evidence that the primary product of the electron transfer is a triplet exciplex. It is thus demonstrated that measuring yields of intersystem crossing processes which accompany electron transfer reactions, can provide a means of obtaining information on the mechanism of the electron transfer process. This method, though indirect, may be of special interest in the case of triplet reactions, since triplet exciplexes are not expected to phosphoresce in liquid solutions, and exciplex absorption might closely re- semble that of the radicals. We expect, however, that these species can be distinguished by their decay times and are planning experiments to detect the exciplex decay on the nanosecond time scale.

\section{Acknowledgement}

We wish to thank Professor H.E.A. Kramer for his support of our work and valuable discussions. The help of Dr.D. Miller and Dr.H. Lee in translating the manuscript is gratefully acknowledged. We thank the Fonds der Chemischen Industrie for their financial assistance.

\section{References}

[1] U. Steiner, G. Winter and H.E.A. Kramer, J. Phys. Chem. 81 (1977) 1104.

[2] K. Schulten, H. Staerk, A. Weller, H.-J. Werner and B. Nickel, Z. Physik. Chem. (Frankfurt) 101 (1976) 371.

[3] M.E. Michcl-Beyerle, R. Haberkorn, W. Bube, E. Steffens, H. Schröder, H.J. Neusser, E.W. Schlag and H. Seidlitz, Chem. Phys. 17 (1976) 139.

[4] A.R. Watkins, Chem. Phys. Letters 43 (1976) 299.

[5] M. Gronkiewicz, B. Kozankiewicz and J. Prochorow, Chem. Phys. Letters 38 (1976) 325;

I. Deperasińska, I. Dresner, B. Kozankiewicz, K. Luczak and J. Prochorow, J. Luminescence, to be published.

[6] W. Broser and H. Fleischhauer, Z. Naturforsch. B 25 (1970) 1389.

[7] D. Rehm and A. Weller, Ber. Bunsenges. Physik. Chem. 73 (1969) 834; Israel J. Chem. 8 (1970) 259.

[8] E. Vogelmann, S. Schreiner, W. Rauscher and H.E.A. Kramer, Z. Physik. Chem. (Frankfurt) 101 (1976) 321.

[9] X. Sasaki, A. Kitani and M. Tsuboi, Nippon Kagaku Kaishi 12 (1973) 2269.

[10] W.R. Carper, R.M. Hedges and A.N. Simpson, J. Phys. Chem. 69 (1965) 1707.

[11] S.P. McGlynn, R. Sunserie and N. Christodouleas, J. Chem. Phys. 37 (1962) 1818.

[12] S.P. McGlynn, T. Azumi and M. Kinoshita, Molecular spectroscopy of the triplet state (Prentice-Hall , Englewood Cliffs, 1969).

[13] J. Friedrich, F. Metz and F. Dörr, Mol. Phys. 30 (1975) 289.

[14] J. Friedrich, G. Weinzzierl and F. Dōrr, Z. Naturforsch. $31 a$ (1976) 748. 